\title{
Order-clustered fixed point theorems on chain-complete preordered sets and their applications to extended and generalized Nash equilibria
}

\author{
Linsen Xie ${ }^{1}$, Jinlu Li² ${ }^{2 *}$ and Wenshan Yang ${ }^{3}$
}

"Correspondence: jli@shawnee.edu

${ }^{2}$ Department of Mathematics, Shawnee State University,

Portsmouth, Ohio 45662, USA Full list of author information is available at the end of the article

\begin{abstract}
In this paper, we introduce the concept of order-clustered fixed point of set-valued mappings on preordered sets and give several generalizations of the extension of the Abian-Brown fixed point theorem provided in (Mas-Colell et al. in Microeconomic Theory, 1995), which is from chain-complete posets to chain-complete preordered sets. By using these generalizations and by applying the order-increasing upward property of set-valued mappings, we prove several existence theorems of the extended and generalized Nash equilibria of nonmonetized noncooperative games on chain-complete preordered sets.
\end{abstract}

MSC: 46B42; 47H10; 58J20; 91A06; 91A10

Keywords: chain-complete preordered sets; order-clustered fixed point; Abian-Brown fixed point theorem; order-increasing upward mapping; nonmonetized noncooperative game; generalized Nash equilibrium; extended Nash equilibrium

\section{Introduction}

In traditional game theory, fixed point theory in topological spaces or metric spaces has been an essential tool for the proof of the existence of Nash equilibria of noncooperative games, in which the payoff functions of the players take real values (see [1-6]). Recently, the concept of nonmonetized noncooperative games has been introduced where the payoff functions of the players take values in ordered sets, on which the topological structure may not be equipped. The existence of generalized and extended Nash equilibria of nonmonetized noncooperative games has been studied by applying fixed point theorems to ordered sets. These games are also named generalized games by some authors (see [713]). Naturally, fixed point theory on ordered sets has revealed its crucial importance in this new subject in game theory.

For single-valued mappings, Tarski provided a fixed point theorem on complete lattices; and Abian and Brown extended it to a fixed point theorem on chain-complete posets (see [14]). In [15], Fujimoto generalized Tarski's fixed point theorem from single-valued mappings to set-valued mappings on complete lattices and gave some applications to vectorcomplementarity problems. Very recently, in [8] and [12], Li provided several versions of extension of both the Abian-Brown fixed point theorem and the Fujimoto-Tarski fixed point theorem to set-valued mappings on chain-complete posets, which are applied to

C) 2013 Xie et al: licensee Springer. This is an Open Access article distributed under the terms of the Creative Commons Attribution License (http://creativecommons.org/licenses/by/2.0), which permits unrestricted use, distribution, and reproduction in any medium, provided the original work is properly cited. 
prove the existence of generalized and extended Nash equilibria of nonmonetized noncooperative games on posets. We list one of them below for easy reference.

Theorem 2.2 in [12] Let $(P, \succcurlyeq)$ be a chain-complete poset and let $F: P \rightarrow 2^{P} \backslash\{\varnothing\}$ be a set-valued mapping. If $F$ satisfies the following three conditions:

A1. $F$ is order-increasing upward;

A2'. $F(x)$ is inductive with a finite number of maximal elements for every $x \in P$;

A3. There is a $y$ in $P$ with $y \preccurlyeq u$ for some $u \in F(y)$.

Then $F$ has a fixed point, that is, there exists $x^{*} \in P$ such that $x^{*} \in F\left(x^{*}\right)$.

In some nonmonetized noncooperative games, both the domains and the ranges of payoff functions may be preordered sets instead of posets; that is, some different elements in the domain or in the range may be order-indifferent or order-equivalent. This is the motivation to consider the fixed point theorems on preordered sets in this paper. This aspect can be more precisely demonstrated by the following example.

For any positive integer $k$, let $\mathbb{R}^{k}$ denote the $k$-dimensional Euclidean space. Let $\succcurlyeq^{k}$ be the binary relation on $\mathbb{R}^{k}$, which is defined as: $x \succcurlyeq^{k} y$ whenever $\|x\| \geq\|y\|$ for $x, y \in \mathbb{R}^{k}$. It is clear that $\succcurlyeq^{k}$ is a preorder relation on $\mathbb{R}^{k}$. Hence $\left(\mathbb{R}^{k}, \succcurlyeq^{k}\right)$ is a preordered set. Then any noncooperative game with all the strategies in $\left(\mathbb{R}^{m}, \succcurlyeq^{m}\right)$ and the values of payoff in $\left(\mathbb{R}^{n}, \succcurlyeq^{n}\right)$, for some positive integers $m$ and $n$, is a nonmonetized noncooperative game, which will be defined in Section 3.

In Section 2 of this paper, we generalize the extensions of the Abian-Brown fixed point theorem provided in [12] from chain-complete posets to chain-complete preordered sets for set-valued mappings. Evidently, they are also generalizations of the Fujimoto-Tarski fixed point theorem from complete lattices to chain-complete preordered sets. In Sections 3 and 4, we apply these generalizations to prove some existence theorems of the extended and generalized Nash equilibria of nonmonetized noncooperative games on chaincomplete preordered sets.

\section{Order-clustered fixed point}

In this section, we recall and provide some concepts and properties of preordered sets, and we introduce the concept of order-clustered fixed point of set-valued mapping on preordered sets. Then we generalize the Abian-Brown fixed point theorem from single-valued mappings to set-valued mappings, which is also from complete lattices to preordered sets. Here we closely follow the notations from [7, 16, 17], and [14].

Let $P$ be a nonempty set. An ordering relation $\succcurlyeq$ on $P$ is said to be a preorder whenever it satisfies the following two conditions:

1. (reflexive) $x \succcurlyeq x$ for every $x \in P$;

2. (transitive) $x \succcurlyeq y$ and $y \succcurlyeq z$ imply $x \succcurlyeq z$ for all $x, y, z \in P$.

Then $P$, together with the preorder $\succcurlyeq$, is called a preordered set, which is denoted by $(P, \succcurlyeq)$. Furthermore, a preorder $\succcurlyeq$ on a preordered set $P$ is said to be a partial order if, in addition to the above two properties, it also satisfies

3. (antisymmetric) $x \succcurlyeq y$ and $y \succcurlyeq x$ imply $x=y$ for every $x, y \in P$. In this case, $(P, \succcurlyeq)$ is simply called a poset. 
Remark 2.1 It is worth mentioning for clarification that a preordered set $(P, \succcurlyeq)$ equipped with the preorder $\succcurlyeq$ on $P$ is defined as a partially ordered system (p.o.s.) in [17].

Let $(P, \succcurlyeq)$ be a preordered set. If $A$ is a subset of $P$, then an element $u$ of $P$ is called an upper bound of $A$ if $x \preccurlyeq u$ for each $x \in A$; if $u \in A$, then $u$ is called a greatest element (or a maximum element) of $A$. The collection of all greatest elements (maximum elements) of $A$ is denoted by $\max A$. A lower bound of $A$ and a smallest element (or a minimum element) of $A$ can be defined similarly. The collection of all smallest elements (minimum elements) of $A$ is denoted by $\min A$. If the set of all upper bounds of $A$ has a smallest element, we call it a supremum of $A$; and the collection of all supremum elements of $A$ is denoted by $\sup A$ or $\vee A$. An infimum of $A$ is similarly defined as a greatest element of the set of all lower bounds of $A$, provided that it exists; and the collection of all infimum elements of $A$ is denoted by $\inf A$ or $\wedge A$.

It is important to note that, from the above definitions, if $A$ is a subset of a preordered set $(P, \succcurlyeq)$, then $\max A, \min A, \sup A$ and $\inf A$ are the subsets of $P$. In particular, if $A$ is a subset of a poset $(P, \succcurlyeq)$, then $\max A, \min A, \sup A$ and $\inf A$ are singletons, provided that they are nonempty. In this case, $\max A, \min A, \sup A$ and $\inf A$ are simply written as the contained elements, respectively.

An element $y \in A$ is said to be a maximal element of the subset $A$ of the preordered set $(P, \succcurlyeq)$ if for any $z \in A$ with $y \preccurlyeq z$ implies $z \preccurlyeq y$. Similar to the definition of maximal element, a minimal element of $A$ can be defined. Then every greatest element (smallest element) of the subset $A$ of the preordered set $(P, \succcurlyeq)$ is a maximal element (minimum element) of $A$; the converse does not hold.

A subset $C$ of the preordered set $(P, \succcurlyeq)$ is said to be totally ordered (or a chain in $P$ ) whenever, for every pair $x, y \in C$, either $x \preccurlyeq y$ or $y \preccurlyeq x$. Following [17] and [4], we have the following definition.

Definition 2.2 A preordered set $(P, \succcurlyeq)$ is said to be

(i) inductive if every totally ordered subset of (chain in) $P$ has an upper bound in $P$;

(ii) chain-complete whenever, for every totally ordered subset $C$ of (a chain in) $P$, the set of all supremum elements of $C$ is a nonempty subset of $P$; that is, $\vee C \neq \varnothing$.

In game theory and decision theory, the decision makers may consider having indifference (same) utilities at some order-equivalent elements in a preordered set. It is the motivation to introduce the following concepts of order-equivalent elements and orderequivalent classes in a preordered set.

Let $\left(P, \succcurlyeq^{P}\right)$ be a preordered set. For any $x, y \in P$, we say that $x, y$ are $\succcurlyeq^{P}$-order equivalent $\left(\succcurlyeq^{P}\right.$-order indifferent), which is denoted by $x \sim^{P} y$, whenever both $x \succcurlyeq^{P} y$ and $y \succcurlyeq^{P} x$ hold. It is clear that $\sim^{P}$ is an equivalence relation on $P$. For any $x \in P$, let $[x]$ denote the order-equivalent class (order-indifferent class) containing $x$, which is called a $\succcurlyeq^{P}$-cluster (or simply an order cluster, or a cluster, if there is no confusion caused). Let $P / \sim^{P}$ or $\tilde{P}$ denote the collection of all order clusters in the preordered set $\left(P, \succcurlyeq^{P}\right)$. So, $x \in[x] \in \tilde{P}$ for every $x \in P$.

Given two preordered sets $\left(X, \succcurlyeq^{X}\right)$ and $\left(U, \succcurlyeq^{U}\right)$, we say that a single-valued mapping $F: X \rightarrow U$ is order-increasing (or order-preserving) if $x \preccurlyeq^{X} y$ in $X$ implies $F(x) \preccurlyeq^{U} F(y)$ in $U$. An order-increasing single-valued mapping $F: X \rightarrow U$ is said to be strictly orderincreasing whenever $x \prec^{X} y$ implies $F(x) \prec^{U} F(x)$. We say that a set-valued mapping $F$ : 
$X \rightarrow 2^{U} \backslash\{\varnothing\}$ is order-increasing upward if $x \preccurlyeq^{X} y$ in $X$ and $u \in F(x)$ imply that there is $w \in F(y)$ such that $u \preccurlyeq^{u} w . F$ is said to be order-increasing downward if $x \preccurlyeq^{X} y$ in $X$ and $w \in F(y)$ imply that there is $u \in F(x)$ such that $u \preccurlyeq^{U} w$. A set-valued mapping $F$ is said to be order-increasing whenever $F$ is order-increasing both upward and downward.

Order-increasing mappings from a preordered set to a preordered set have the following equivalent classes preserving property.

Lemma 2.3 Let $\left(X, \succcurlyeq^{X}\right)$ and $\left(U, \succcurlyeq^{U}\right)$ be two preordered sets and let $F: X \rightarrow U$ be an orderincreasing single-valued mapping. Then, for every $x$ in $X, y \in[x]$ implies $F(y) \in[F(x)]$ in $U$.

Proof Without any confusion, $[x]$ is understood to be an order-equivalence class in $\left(X, \succcurlyeq^{X}\right)$ with respect to the preorder $\succcurlyeq^{X}$ and $[F(x)]$ is understood to be an order-equivalence class in $\left(U, \succcurlyeq^{U}\right)$ with respect to the preorder $\succcurlyeq^{U}$. For every $x$ in $X, y \in[x]$ if and only if both of $x \preccurlyeq^{X} y$ and $y \preccurlyeq^{X} x$ hold in $X$. Then the order-increasing property of $F$ implies that both of $F(x) \preccurlyeq^{U} F(y)$ and $F(y) \preccurlyeq^{U} F(x)$ hold in $U$. That is, $F(y) \in[F(x)]$.

In game theory and decision theory, there are useful mappings $F: X \rightarrow U$ between two preordered sets which map order-indifferent elements in $X$ to order-indifferent elements in $U$. That is, if the inputs of the mapping $F$ are $\succcurlyeq^{X}$-order equivalent elements in $X$, then the outputs of $F$ are $\succcurlyeq^{U}$-order equivalent elements in $U$. This leads us to the following definition.

Definition 2.4 Let $\left(X, \succcurlyeq^{X}\right)$ and $\left(U, \succcurlyeq^{U}\right)$ be two preordered sets and let $F: X \rightarrow U$ be a single-valued mapping. $F$ is said to be order cluster-preserving, whenever

$$
x \sim^{X} y \text { implies } F(x) \sim^{U} F(y) \text { for } x, y \in X .
$$

Lemma 2.5 Every order-preserving single-valued mapping from a preordered set to a preordered set is cluster-preserving.

Proof The order cluster-preserving property (2.1) immediately follows from Lemma 2.3.

Definition 2.6 Let $\left(X, \succcurlyeq^{X}\right)$ be a preordered set and let $F: X \rightarrow 2^{X} \backslash\{\varnothing\}$ be a set-valued mapping. An element $x \in X$ is called

1. a fixed point of $F$, whenever $x \in F(x)$;

2. an $\succcurlyeq^{X}$-clustered fixed point (an order-clustered fixed point, or simply a clustered fixed point) of $F$, whenever there is a $w \in[x]$ such that $w \in F(x)$.

Definition 2.6 can be explained as follows: an element $x \in X$ is an order-clustered fixed point of $F$, whenever there is a $w \in X$ with $w \sim^{X} x$ such that $w \in F(x)$. Since $x \sim^{X} x$, then we have the following property:

$$
x \text { is a fixed point of } F \Rightarrow x \text { is an order-clustered fixed point of } F \text {. }
$$

It is clear that the inverse of the above implication does not hold. Hence, order-clustered fixed points are generalizations of the fixed points. It is important to notice that if $\left(X, \succcurlyeq^{X}\right)$ is a poset, then an order-clustered fixed point coincides with a fixed point. 
Let $(P, \succcurlyeq)$ be a chain-complete preordered set and let $F: P \rightarrow 2^{P} \backslash\{\varnothing\}$ be a set-valued mapping. Following Fujimoto [15], we have the following notation:

$$
S F(x)=\{z \in P: z \preccurlyeq u \text { for some } u \in F(x)\} \quad \text { for every } x \in P .
$$

Theorem 2.7 Let $(P, \succcurlyeq)$ be a chain-complete preordered set and let $F: P \rightarrow 2^{P} \backslash\{\varnothing\}$ be a set-valued mapping. If $F$ satisfies the following three conditions:

A1. $F$ is order-increasing upward;

A2. $S F(x)$ is an inductive subset of $P$ for each $x \in P$;

A3. There is a $y$ in $P$ with $y \preccurlyeq u$ for some $u \in F(y)$.

Then $F$ has an order-clustered fixed point, that is, there exists $x^{*} \in P$ with $w \in\left[x^{*}\right]$ such that $w \in F\left(x^{*}\right)$.

Proof Let

$$
A=\{x \in P: x \preccurlyeq u \text { for some } u \in F(x)\} .
$$

It is clear that the element $y$ given in assumption $\mathrm{A} 3$ is in $A$; and therefore $A \neq \varnothing$. Very similarly to the proof of the extension of Tarski's fixed point theorem by Fujimoto in [15], by applying isotonic property $\mathrm{A} 1$ and assumption $\mathrm{A} 2$ in this theorem, we can show that the set $A$ is inductive. To this end, take an arbitrary totally ordered subset (a chain) $C \subseteq A$. Since $C$ is also a chain of the chain-complete preordered set $(P, \succcurlyeq)$, then $\vee C \neq \varnothing$ and $\vee C \subseteq P$. Take an arbitrary element $b \in \vee C$. So, for any $x \in C \subseteq A$, from (2.3), there is a $u \in F(x)$ with $x \preccurlyeq u$. On the other hand, from $x \preccurlyeq b$ and $u \in F(x)$, applying assumption A1, there is $v \in F(b)$ such that $x \preccurlyeq u \preccurlyeq v$; and hence $x \in S F(b)$ for every $x \in C$. It implies

$$
C \subseteq S F(b)
$$

From assumption A2, $S F(b)$ is inductive; and therefore the totally ordered subset (chain) $C$ of $S F(b)$ has an upper bound in $S F(b)$, say $c$, with $c \in S F(b)$. From (2.2), it yields that there is a $u \in F(b)$ such that $c \preccurlyeq u$.

Since $b \in \vee C$ and $c$ is an upper bound of $C$, we have $b \preccurlyeq c$. Then we obtain

$$
b \preccurlyeq c \preccurlyeq u \quad \text { for some } u \in F(b) \text {, }
$$

which implies that $b \in A$. Hence $b$ is an upper bound of the given chain $C$ in $A$; and therefore $A$ is inductive.

Then applying Zorn's lemma (Theorem I.2.7 in [17]), the inductive set $A$ has a maximal element, say $x^{*} \in A$. Equation (2.3) implies that there is $w \in F\left(x^{*}\right)$ such that $x^{*} \preccurlyeq w$. For this element $w \in F\left(x^{*}\right)$ with $x^{*} \preccurlyeq w$, from assumption A1 in this theorem, there is $z \in F(w)$ with $w \preccurlyeq z$, which implies that $w \in A$. Since $x^{*}$ is a maximal element of $A$, from $x^{*} \preccurlyeq w$, we must have $x^{*} \sim w \in F\left(x^{*}\right)$. Hence, $x^{*}$ is an order-clustered fixed point of $F$. This theorem is proved.

Theorem 2.8 Let $(P, \succcurlyeq)$ be a chain-complete preordered set and let $F: P \rightarrow 2^{P} \backslash\{\varnothing\}$ be a set-valued mapping. If $F$ satisfies conditions $\mathrm{A} 1$ and $\mathrm{A} 3$ given in Theorem 2.7 and 
A2'. $F(x)$ is inductive with a finite number of maximal element clusters for every $x \in P$,

then F has an order-clustered fixed point.

Proof Take the same set $A=\{x \in P: x \preccurlyeq u$ for some $u \in F(x)\}$ as defined in the proof of Theorem 2.7. Assumption A3 in this theorem also implies $A \neq \varnothing$. Then we show that $A$ is inductive. To this end, take an arbitrary chain $C$ of $A$. Since $(P, \succcurlyeq)$ is a chain-complete preordered set, then $\vee C \neq \varnothing$ and $\vee C \subseteq P$. Pick an arbitrary element $b \in \vee C$. For every $x \in C \subseteq A$, there is a point $u_{x} \in F(x)$ satisfying $x \preccurlyeq u_{x}$. On the other hand, from $x \preccurlyeq b$, assumption $\mathrm{A} 1$ implies that there is an $e(x) \in F(b)$ such that $u_{x} \preccurlyeq e(x)$. Then we obtain a mapping $e: C \rightarrow F(b)$ satisfying the following order inequality:

$$
x \preccurlyeq u_{x} \preccurlyeq e(x) \quad \text { with } u_{x} \in F(x) \text { and } e(x) \in F(b) \text { for every } x \in C \text {. }
$$

From assumption $\mathrm{A}^{\prime}$ in this theorem, we can write the set of maximal element clusters of $F(b)$ by $\left\{\left[u_{1}\right],\left[u_{2}\right], \ldots,\left[u_{m}\right]\right\} \subseteq 2^{F(b)}$, for some positive integer $m$, with $\left\{u_{1}, u_{2}, \ldots, u_{m}\right\} \subseteq$ $F(b)$. We claim that there are elements $x_{0} \in C$ and $u_{j}$, for some $j$ with $1 \leq j \leq m$, such that

$$
x \preccurlyeq u_{j} \quad \text { for all } x \in C \text { with } x \succcurlyeq x_{0} .
$$

In fact, from (2.4), we have, for every $x \in C, x \preccurlyeq e(x) \in F(\vee C)$. Since $F(b)$ is inductive and $\left\{\left[u_{1}\right],\left[u_{2}\right], \ldots,\left[u_{m}\right]\right\}$ is the set of all the maximal element clusters of $F(b)$, then, for any given $x \in C$, we must have

$$
x \preccurlyeq e(x) \preccurlyeq u_{i} \quad \text { for some } i \text { with } 1 \leq i \leq m \text {. }
$$

By applying (2.6), the proof of (2.5) is similar to the proof of Theorem 2.2 in [12]. Then, from the claim (2.5), we have $b \preccurlyeq u_{j} \in F(b)$ for some $j$ with $1 \leq j \leq m$; and hence $b \in A$. This implies that $C$ has an upper bound $b$ in $A$; and therefore, $A$ is inductive. The rest of the proof is very similar to the proof of Theorem 2.7.

As a consequence of Theorem 2.8, we obtain the following special cases of the extensions of the Abian-Brown fixed point theorem in preordered sets.

Corollary 2.9 Let $(P, \succcurlyeq)$ be a chain-complete preordered set and let $F: P \rightarrow 2^{P} \backslash\{\varnothing\}$ be a set-valued mapping. If $F$ satisfies conditions $\mathrm{A} 1$ and $\mathrm{A} 3$ given in Theorem 2.7 and

A2". $F(x)$ has a maximum element for every $x \in P$,

then F has an order-clustered fixed point.

If we consider some special cases of the chain-complete preordered set $(P, \succcurlyeq)$ for which condition A3 can be automatically satisfied, then we obtain the following corollaries that can also be considered as extensions of both the Fujimoto-Tarski fixed point theorem from complete lattices to a chain-complete preordered set $\backslash$ and the Abian-Brown fixed point theorem from single-valued mappings to set-valued mappings on chain-complete preordered sets. 
Corollary 2.10 Let $(P, \succcurlyeq)$ be a chain-complete preordered set with $\wedge P \neq \varnothing$ in $P$. Let $F$ : $P \rightarrow 2^{P} \backslash\{\varnothing\}$ be a set-valued mapping. If $F$ satisfies condition A1 given in Theorem 2.7 and one of conditions A2, A2', or A2" given in Theorems 2.7, 2.8 and Corollary 2.9, respectively, then $F$ has an order-clustered fixed point.

Proof Take $y \in \wedge P$ in $P$, then for any $u \in F(y)$, we obviously have $y \preccurlyeq u$. So, $y \in \wedge P$ satisfies assumption A3 in Theorem 2.7.

As consequences, when posets are considered as special preordered sets, the extensions of the Abian-Brown fixed point theorem in posets provided in [12] immediately follow from Corollary 2.10. As a matter of fact, we have the following result on a chain-complete poset, which is more general than the extension of Tarski's fixed point theorem on complete lattice by Fujimoto [15].

Corollary 2.11 Let $(P, \succcurlyeq)$ be a chain-complete poset with $\wedge P$ exists in $P$. Let $F: P \rightarrow$ $2^{P} \backslash\{\varnothing\}$ be a set-valued map. If F satisfies condition A1 given in Theorem 2.7 and one of conditions A2, A2', or A2" given in Theorems 2.7, 2.8, and Corollary 2.9, respectively, then $F$ has a fixed point.

\section{Applications to the extended Nash equilibria of nonmonetized noncooperative games on chain-complete preordered sets}

In this section, we recall the concepts of the nonmonetized noncooperative games and the generalized and extended Nash equilibria of these games on preordered sets, which were studied in [8-13]. Then we apply the extensions of the Abian-Brown fixed point theorem provided in the last section to prove some existence theorems for the extended Nash equilibrium of nonmonetized noncooperative games on chain-complete preordered sets, which can be considered as extensions of the results proved by Li in [12], which are on chain-complete posets.

Definition 3.1 Let $n$ be a positive integer greater than 1 . An $n$-person nonmonetized noncooperative game consists of the following elements:

1. the set of $n$ players, which is denoted by $N=\{1,2,3, \ldots, n\}$;

2. the collection of $\mathrm{n}$ strategy sets $\left\{S_{1}, S_{2}, \ldots, S_{n}\right\}$, for the $n$ players respectively, such that $\left(S_{i}, \succcurlyeq_{i}\right)$ is a chain-complete preordered set for player $i=1,2,3, \ldots, n$, with notation $S=S_{1} \times S_{2} \times \cdots \times S_{n}$

3. the outcome space $\left(U ; \succcurlyeq^{U}\right)$ that is a preordered set;

4. the $n$ payoff functions $f_{1}, f_{2}, \ldots, f_{n}$, where $f_{i}$ is the payoff function for the player $i$ that is a mapping from $S_{1} \times S_{2} \times \cdots \times S_{n}$ to the preordered set $\left(U ; \succcurlyeq^{U}\right)$, for $i=1,2,3, \ldots, n$. We define $f=\left\{f_{1}, f_{2}, \ldots, f_{n}\right\}$.

This game is denoted by $\Gamma=(N, S, f, U)$.

The rule to play in an $n$-person nonmonetized noncooperative game $\Gamma=(N, S, f, U)$ is that when all $n$ players $1,2,3, \ldots, n$ simultaneously and independently choose their own strategies $x_{1}, x_{2}, \ldots, x_{n}$, where $x_{i} \in S_{i}$, for $i=1,2,3, \ldots, n$, then the player $i$ will receive his or her utility (payoff) $f_{i}\left(x_{1}, x_{2}, \ldots, x_{n}\right) \in U$. For any $x=\left(x_{1}, x_{2}, \ldots, x_{n}\right) \in S$, and for every given $i=1,2,3, \ldots, n$, as usual, we define

$$
x_{-i}:=\left(x_{1}, x_{2}, \ldots, x_{i-1}, x_{i+1}, \ldots, x_{n}\right) \quad \text { and } \quad S_{-i}:=S_{1} \times S_{2} \times \cdots \times S_{i-1} \times S_{i+1} \times \cdots \times S_{n} .
$$


Then $x_{-i} \in S_{-i}$ and $x$ can simply be written as $x=\left(x_{i}, x_{-i}\right)$. Moreover, we define

$$
f_{i}\left(S_{i}, x_{-i}\right)=\left\{f_{i}\left(t_{i}, x_{-i}\right): t_{i} \in S_{i}\right\}
$$

An $n$-person nonmonetized noncooperative game defined in this section is also called a generalized game (see [7]). Now we recall the extensions of the concept of Nash equilibrium of noncooperative games to the generalized Nash equilibrium and the extended Nash equilibrium of nonmonetized noncooperative games.

Definition 3.2 In an $n$-person nonmonetized noncooperative game $\Gamma=(N, S, f, U)$, a selection of strategies $\left(\widehat{x}_{1}, \widehat{x}_{2}, \ldots, \widehat{x}_{n}\right) \in S_{1} \times S_{2} \times \cdots \times S_{n}$ is called

1. a generalized Nash equilibrium of this game if, for every $i=1,2,3, \ldots, n$, the following order inequality holds:

$$
f_{i}\left(x_{i}, \widehat{x}_{-i}\right) \preccurlyeq f_{i}\left(\widehat{x}_{i}, \widehat{x}_{-i}\right) \quad \text { for all } x_{i} \in S_{i} ;
$$

2. an extended Nash equilibrium of this game if, for every $i=1,2,3, \ldots, n$, the following order inequality holds:

$$
f_{i}\left(x_{i}, \widehat{x}_{-i}\right) \nsucc^{U} f_{i}\left(\widehat{x}_{i}, \widehat{x}_{-i}\right) \quad \text { for all } x_{i} \in S_{i}
$$

It is clear that any generalized Nash equilibrium of an $n$-person nonmonetized noncooperative game is an extended Nash equilibrium of this game; and the converse may not be true.

Lemma 3.3 Let $\left(S_{i}, \succcurlyeq_{i}\right)$ be a preordered set for every $i=1,2, \ldots, n$. Let $\succcurlyeq^{S}$ be the coordinate ordering on $S$ induced by the preorders $\succcurlyeq_{i}$, that is, for any $x, y \in S$ with $x=\left(x_{1}, x_{2}, \ldots, x_{n}\right)$ and $y=\left(y_{1}, y_{2}, \ldots, x_{n}\right)$,

$$
x \succcurlyeq^{S} y \quad \text { if and only if } \quad x_{i} \succcurlyeq_{i} y_{i} \quad \text { for all } i=1,2, \ldots, n \text {. }
$$

Then $\succcurlyeq^{S}$ is a preorder on $S$; and hence $\left(S, \succcurlyeq^{S}\right)$ is a preordered set. Furthermore, if every $\left(S_{i}, \succcurlyeq_{i}\right)$ is chain-complete (inductive), then $\left(S, \succcurlyeq^{S}\right)$ is also chain-complete (inductive). Moreover, we have

$$
\tilde{S}=\tilde{S}_{1} \times \tilde{S}_{2} \times \cdots \times \tilde{S}_{n}
$$

Furthermore, for any $x=\left(x_{1}, x_{2}, \ldots, x_{n}\right) \in S$, we have $[x]=\left(\left[x_{1}\right],\left[x_{2}\right], \ldots,\left[x_{n}\right]\right)$, where $[x]$ stands for an $\succcurlyeq^{S}$-cluster in $\tilde{S}$ and $\left[x_{1}\right],\left[x_{2}\right], \ldots,\left[x_{n}\right]$ are order clusters in $\tilde{S}_{1}, \tilde{S}_{2}, \ldots, \tilde{S}_{n}$, respectively.

Proof The proof is straightforward and is omitted here.

Let $A$ be a subset of a preordered set $(P, \succcurlyeq)$. The following subset of $P$ is called the $\succcurlyeq$-downward set of $A$ denoted by $D(A)$,

$$
D(A)=\{z \in P: z \preccurlyeq u \text { for some } u \in A\} .
$$


The following theorem provides a result for the existence of an extended Nash equilibrium of nonmonetized noncooperative games on preordered sets.

Theorem 3.4 Let $\Gamma=(N, S, f, U)$ be an n-person nonmonetized noncooperative game. Suppose that for every player $i=1,2,3, \ldots, n$, and for any $x \in S, f_{i}: S \rightarrow U$ is a clusterpreserving single-valued mapping that satisfies the following conditions:

G1. $f_{i}\left(S_{i}, x_{-i}\right)$ is an inductive subset of the preordered set $\left(U, \succcurlyeq^{U}\right)$;

G2. The $\succcurlyeq_{i}$-downward set of the inverse image $\left\{z_{i} \in S_{i}: f_{i}\left(z_{i}, x_{-}\right)\right.$is a maximal element of $\left.f_{i}\left(S_{i}, x_{-i}\right)\right\}$ is an inductive subset of $S_{i}$;

G3. For $x \preccurlyeq^{S} y$ in $S$, if $z_{i} \in S_{i}$ with $f_{i}\left(z_{i}, x_{-i}\right)$ is a maximal element of $f_{i}\left(S_{i}, x_{-i}\right)$, then there is $w_{i} \in S_{i}$ with $z_{i} \preccurlyeq_{i} w_{i}$ such that $f_{i}\left(w_{i}, y_{-i}\right)$ is a maximal element of $f_{i}\left(S_{i}, y_{-i}\right)$.

If there are $p=\left(p_{i}, p_{-i}\right), q=\left(q_{i}, q_{-i}\right) \in S$ satisfying that

$$
p \preccurlyeq^{S} q \text { and } f_{i}\left(q_{i}, p_{-i}\right) \text { is a maximal element of } f_{i}\left(S_{i}, p_{-i}\right) \text {, }
$$

then this game $\Gamma$ has an extended Nash equilibrium $\widehat{x}=\left(\widehat{x}_{1}, \widehat{x}_{2}, \ldots, \widehat{x}_{n}\right)$. Moreover, every element in $[\widehat{x}]$ is an extended Nash equilibrium of $\Gamma$.

Proof The first part of the proof is similar to the proof of Theorem 3.4 in [12]. Since $\left(S_{i}, \succcurlyeq_{i}\right)$ is a chain-complete preordered set for every $i=1,2, \ldots, n$, then from Lemma $3.3,\left(S, \succcurlyeq^{S}\right)$ is also a chain-complete preordered set equipped with the product order $\succcurlyeq^{S}$.

For every fixed $i=1,2,3, \ldots, n$, we define a set-valued mapping $T_{i}: S \rightarrow 2^{S_{i}} \backslash\{\varnothing\}$ by

$$
\begin{aligned}
& T_{i}(x)=\left\{z_{i} \in S_{i}: f_{i}\left(z_{i}, x_{-i}\right) \text { is a maximal element of } f_{i}\left(S_{i}, x_{-i}\right)\right\} \\
& \text { for all } x=\left(x_{1}, x_{2}, \ldots, x_{n}\right) \in S .
\end{aligned}
$$

From assumption G1 of this theorem, for every fixed element $x \in S, f_{i}\left(S_{i}, x_{-i}\right)$ is an inductive subset of $U$. By applying Zorn's lemma, the range $f_{i}\left(S_{i}, x_{-i}\right)$ has a maximal element; and therefore $T_{i}(x)$ is a nonempty subset of $S_{i}$. Hence, the map $T_{i}: S \rightarrow 2^{S_{i}} \backslash\{\varnothing\}$ is well defined. Then we define a set-valued mapping $T: S \rightarrow 2^{S} \backslash\{\varnothing\}$ by

$$
T(x)=T_{1}(x) \times T_{2}(x) \times \cdots \times T_{n}(x) \quad \text { for all } x \in S .
$$

Now we prove that the mapping $T$ satisfies all conditions A1, A2 and A3 in the first extension of the Abian-Brown fixed point theorem in a chain-complete preordered set provided in the preceding section.

At first, we show that $T$ satisfies assumption A1: $T$ is $\succcurlyeq^{S}$-increasing upward. For any given $x \preccurlyeq^{S} y$ in $S$ and for any $z=\left(z_{1}, z_{2}, \ldots, z_{n}\right) \in T(x)$, for every $i=1,2, \ldots, n$, we have $z_{i} \in T_{i}(x)$, that is, $f_{i}\left(z_{i}, x_{-i}\right)$ is a maximal element of $f_{i}\left(S_{i}, x_{-i}\right)$. Then from hypothesis G3 of this theorem, there is $w_{i} \in S_{i}$ with $z_{i} \preccurlyeq_{i} w_{i}$ such that $f_{i}\left(w_{i}, y_{-i}\right)$ is a maximal element of $f_{i}\left(S_{i}, y_{-i}\right)$, that is, $w_{i} \in T_{i}(y)$. Take $w=\left(w_{1}, w_{2}, \ldots, w_{n}\right)$. We obtain that $z \preccurlyeq^{S} w$ and $w \in T(y)$. Hence, $T$ is isotone.

Secondly, we prove that the mapping $T$ satisfies assumption A2 in Theorem 2.7. For an arbitrary $x$ in $S$, with respect to the mapping $T$ and from (2.2), we write

$$
S T(x)=\left\{t \in S: t \preccurlyeq^{S} z \text { for some } z \in T(x)\right\} .
$$


For every $i=1,2, \ldots, n$, with respect to the mapping $T_{i}$, we have

$$
\begin{aligned}
S T_{i}(x)= & \left\{t_{i} \in S_{i}: t_{i} \preccurlyeq_{i} z_{i} \text { for some } z_{i} \in T_{i}(x)\right\} \\
= & \left\{t_{i} \in S_{i}: t_{i} \preccurlyeq_{i} z_{i} \text { for some } z_{i} \in S_{i} \text { such that } f_{i}\left(z_{i}, x_{-i}\right)\right. \\
& \text { is a maximal element of } \left.f_{i}\left(S_{i}, x_{-i}\right)\right\} \\
= & D\left(\left\{z_{i} \in S_{i}: f_{i}\left(z_{i}, x_{-i}\right) \text { is a maximal element of } f_{i}\left(S_{i}, x_{-i}\right)\right\}\right) .
\end{aligned}
$$

From assumption G2 of this theorem, the $\succcurlyeq_{i}$-downward set of $\left\{z_{i} \in S_{i}: P_{i}\left(z_{i}, x_{-i}\right)\right.$ is a maximal element of $\left.P_{i}\left(S_{i}, x_{-i}\right)\right\}$ is inductive, which implies that $S T_{i}(x)$ is an inductive subset of $S$, so is $S T(x)$.

Finally, for the given elements $p, q \in S$ in this theorem, from condition (3.1), we have $p \preccurlyeq^{S} q$ and $f_{i}\left(q_{i}, p_{-i}\right)$ is a maximal element of $f_{i}\left(S_{i}, p_{-i}\right)$. This implies that $q \in T(p)$ with $p \preccurlyeq^{S} q$; and hence the mapping $T$ satisfies assumption A3.

Thus the mapping $T: S \rightarrow 2^{S} \backslash\{\varnothing\}$ satisfies all conditions A1, A2 and A3 in Theorem 2.7. Since $S$ is a chain-complete preordered set, applying Theorem 2.7, $T$ has an orderclustered fixed point $\widehat{x}=\left(\widehat{x}_{1}, \widehat{x}_{2}, \ldots, \widehat{x}_{n}\right) \in S$. Hence, there is $\hat{t} \in[\widehat{x}]$ such that $\hat{t} \in T(\widehat{x})$. This implies $\widehat{t}_{i} \in T_{i}(\widehat{x})$; that is,

$$
f_{i}\left(\widehat{t}_{i}, \widehat{x}_{-i}\right) \text { is a maximal element of } f_{i}\left(S_{i}, \widehat{x}_{-i}\right) \text { for every fixed } i=1,2, \ldots, n \text {. }
$$

For every $i=1,2,3, \ldots, n$, it is equivalent to

$$
f_{i}\left(t_{i}, \widehat{x}_{-i}\right) \nsucc^{U} f_{i}\left(\hat{t}_{i}, \widehat{x}_{-i}\right) \quad \text { for all } t_{i} \in S_{i} \text {. }
$$

The following is from the definition of the product order $\succcurlyeq^{S}$ on $\left(S, \succcurlyeq^{S}\right)$ :

$$
\widehat{t} \sim^{S} \widehat{x} \Rightarrow \hat{t}_{i} \sim_{i} \widehat{x}_{i} \Rightarrow\left(\hat{t}_{i}, \widehat{x}_{-i}\right) \sim^{S}\left(\widehat{x}_{i}, \widehat{x}_{-i}\right)=\widehat{x} \quad \text { for } i=1,2, \ldots, n .
$$

Then from (3.3), $\hat{t} \in[\widehat{x}]$ implies $\left(\widehat{t}_{i}, \widehat{x}_{-i}\right) \sim^{S}\left(\widehat{x}_{i}, \widehat{x}_{-i}\right)$ for $i=1,2, \ldots, n$. Since the payoff function $f_{i}: S \rightarrow U$ is a cluster-preserving single-valued mapping, from $\left(\widehat{t}_{i}, \widehat{x}_{-i}\right) \sim^{S}\left(\widehat{x}_{i}\right.$, $\hat{x}_{-i}$ ) and Definition 2.4, it yields that

$$
f_{i}\left(\widehat{t}_{i}, \widehat{x}_{-i}\right) \in\left[f_{i}\left(\widehat{x}_{i}, \widehat{x}_{-i}\right)\right] ; \quad \text { that is, } \quad f_{i}\left(\widehat{t}_{i}, \widehat{x}_{-i}\right) \sim^{U} f_{i}\left(\widehat{x}_{i}, \widehat{x}_{-i}\right) .
$$

Combining (3.2) and (3.4), we have

$$
f_{i}\left(t_{i}, \widehat{x}_{-i}\right) \nsucc^{U} f_{i}\left(\widehat{x}_{i}, \widehat{x}_{-i}\right) \quad \text { for all } t_{i} \in S_{i} \text {. }
$$

This shows that $\widehat{x}=\left(\widehat{x}_{1}, \widehat{x}_{2}, \ldots, \widehat{x}_{n}\right)$ is an extended Nash equilibrium of this game. With the same argument as above, we can show that, for any $\widehat{z} \in[\widehat{x}], f_{i}\left(\bar{z}_{i}, \bar{z}_{-i}\right) \sim^{U} f_{i}\left(\widehat{x}_{i}, \widehat{x}_{-i}\right)$ holds. Then, repeating the argument from (3.2) to (3.5), we obtain that $\bar{z}$ is also an extended Nash equilibrium of this game. This completes the proof of this theorem.

We can apply Theorem 2.8, which is a different version of the extensions of the AbianBrown fixed point theorem on preordered sets, to similarly show the following result. We list it as a theorem without giving the proof. 
Theorem 3.5 Let $\Gamma=(N, S, f, U)$ be an n-person nonmonetized noncooperative game. Suppose that for every player $i=1,2,3, \ldots, n, f_{i}: S \rightarrow U$ is a cluster-preserving single-valued mapping. If, for any $x \in S$ in addition to that, $f_{i}$ satisfies assumptions G1, G3, and condition (3.1) given in Theorem 3.4, $f_{i}$ also satisfies the following condition:

G2'. The inverse image $\left\{z_{i} \in S_{i}: P_{i}\left(z_{i}, x_{-i}\right)\right.$ is a maximal element of $\left.P_{i}\left(S_{i}, x_{-i}\right)\right\}$ is inductive with a finite number of maximal element clusters for each $x \in S$.

Then there is an $\succcurlyeq^{S}$-cluster in $S$, in which every element is an extended Nash equilibrium of $\Gamma$.

In case the condition $\wedge S_{i} \neq \varnothing$ holds for the strategy set $\left(S_{i}, \succcurlyeq_{i}\right)$, for every $i$, condition (3.1) in Theorems 3.4 and 3.5 can be removed. As a consequence of Theorem 3.5, we have the following.

Corollary 3.6 Let $\Gamma=(N, S, f, U)$ be an n-person nonmonetized noncooperative game. Suppose that for every player $i=1,2,3, \ldots, n, \wedge S_{i} \neq \varnothing$ and for any $x \in S, f_{i}: S \rightarrow U$ is a cluster-preserving single-valued mapping. If, for any $x \in S$ in addition to that, $f_{i}$ satisfies assumptions $\mathrm{G} 1$ and $\mathrm{G} 3, f_{i}$ also satisfies one of assumptions $\mathrm{G} 2$ or G2' given in Theorem 3.4 and Theorem 3.5, then there is an $\succcurlyeq^{S}$-cluster in $S$, in which every element is an extended Nash equilibrium of $\Gamma$.

Proof Take $p \in \wedge S$ and let $q$ be a maximum element of $F(p)$. It is clear that $p$ and $q$ satisfy condition (3.1) given in Theorem 3.4.

\section{Applications to the generalized Nash equilibria of nonmonetized noncooperative games on chain-complete preordered sets}

It is clear that for $\left(t_{i}, \widehat{x}_{-i}\right),\left(\widehat{x}_{i}, \widehat{x}_{-i}\right) \in S$, the order inequality $f_{i}\left(t_{i}, \widehat{x}_{-i}\right) \preccurlyeq^{u} f_{i}\left(\widehat{x}_{i}, \widehat{x}_{-i}\right)$ implies $f_{i}\left(t_{i}, \widehat{x}_{-i}\right) \chi^{U} f_{i}\left(\widehat{x}_{i}, \widehat{x}_{-i}\right)$. Hence, the generalized Nash equilibria can be considered as special cases of the extended Nash equilibria of $n$-person nonmonetized noncooperative games. The conditions for the existence of a generalized Nash equilibrium of an $n$-person nonmonetized noncooperative game should be stronger than the conditions for the existence of an extended Nash equilibrium applied to the theorems in the preceding section.

Theorem 4.1 Let $\Gamma=(N, S, f, U)$ be an n-person nonmonetized noncooperative game. Suppose that for every player $i=1,2,3, \ldots, n, f_{i}: S \rightarrow U$ is a cluster-preserving single-valued mapping, and for any $x \in S$, in addition to that, $f_{i}$ satisfies the following two conditions:

g1. $f_{i}\left(S_{i}, x_{-i}\right)$ is a subset of $\left(U, \succcurlyeq^{U}\right)$ with a maximum element;

g3. For $x \preccurlyeq^{S} y$ in $S$, if $z_{i} \in S_{i}$ with $f_{i}\left(z_{i}, x_{-i}\right)$ is a maximum element of $f_{i}\left(S_{i}, x_{-i}\right)$, then there is $w_{i} \in S_{i}$ with $z_{i} \preccurlyeq_{i} w_{i}$ such that $f_{i}\left(w_{i}, y_{-i}\right)$ is a maximum element of $f_{i}\left(S_{i}, y_{-i}\right)$,

$f_{i}$ also satisfies one of the following conditions:

g2. The $\succcurlyeq_{i}$-downward set of the inverse image $\left\{z_{i} \in S_{i}: P_{i}\left(z_{i}, x_{-i}\right)\right.$ is a maximum element of $\left.f_{i}\left(S_{i}, x_{-}\right)\right\}$is an inductive subset of $S_{i}$;

$\mathrm{g2}^{\prime}$. The inverse image $\left\{z_{i} \in S_{i}: P_{i}\left(z_{i}, x_{-}\right)\right.$is a maximum element of $\left.f_{i}\left(S_{i}, x_{-}\right)\right\}$is inductive with a finite number of maximal element clusters for each $x \in S$.

If there are $p=\left(p_{i}, p_{-i}\right), q=\left(q_{i}, q_{-i}\right) \in S$ satisfying that

$$
p \preccurlyeq^{S} q \text { and } f_{i}\left(q_{i}, p_{-i}\right) \text { is a maximum element of } f_{i}\left(S_{i}, p_{-i}\right) \text {, }
$$


then there is an $t^{S}$-cluster in $S$, in which every element is a generalized Nash equilibrium of $\Gamma$.

Proof The proof of this theorem is similar to the proof of Theorem 3.4. For every fixed $i=1,2,3, \ldots, n$, we define a mapping $T_{i}: S \rightarrow 2^{S_{i}} \backslash\{\varnothing\}$ by

$$
\begin{aligned}
& T_{i}(x)=\left\{z_{i} \in S_{i}: f_{i}\left(z_{i}, x_{-i}\right) \text { is a maximum element of } f_{i}\left(S_{i}, x_{-i}\right)\right\} \\
& \text { for all } x=\left(x_{1}, x_{2}, \ldots, x_{n}\right) \in S .
\end{aligned}
$$

We also define the product mapping $T: S \rightarrow 2^{S} \backslash\{\varnothing\}$ by

$$
T(x)=T_{1}(x) \times T_{2}(x) \times \cdots \times T_{n}(x) \quad \text { for all } x \in S .
$$

Then, similarly to the proof of Theorem 3.4, we can show that the mapping $T$ has an orderclustered fixed point $\widehat{x}=\left(\widehat{x}_{1}, \widehat{x}_{2}, \ldots, \widehat{x}_{n}\right) \in S$. Hence, there is $\bar{t} \in[\widehat{x}]$ such that $\bar{t} \in T(\widehat{x})$. This implies $\hat{t}_{i} \in T_{i}(\hat{x})$; that is,

$$
f_{i}\left(\widehat{t}_{i}, \widehat{x}_{-i}\right) \text { is a maximum element of } f_{i}\left(S_{i}, \widehat{x}_{-i}\right) \text { for every fixed } i=1,2, \ldots, n \text {. }
$$

It is equivalent to the following: for every $i=1,2,3, \ldots, n$, we have

$$
f_{i}\left(t_{i}, \widehat{x}_{-i}\right) \preccurlyeq^{U} f_{i}\left(\widehat{t}_{i}, \widehat{x}_{-i}\right) \quad \text { for all } t_{i} \in S_{i},
$$

From (3.3), $\bar{t} \sim^{S} \widehat{x}$ implies $\left(\widehat{t}_{i}, \widehat{x}_{-i}\right) \sim^{S}\left(\widehat{x}_{i}, \widehat{x}_{-i}\right)$ for every fixed $i=1,2, \ldots, n$. Since $f_{i}: S \rightarrow$ $U$ is a cluster-preserving single-valued mapping from (2.1) in Definition 2.4, it implies

$$
f_{i}\left(\hat{t}_{i}, \widehat{x}_{-i}\right) \sim^{U} f_{i}\left(\widehat{x}_{i}, \widehat{x}_{-i}\right) .
$$

Combining (4.1) and (4.2), we have

$$
f_{i}\left(t_{i}, \widehat{x}_{-i}\right) \preccurlyeq^{U} f_{i}\left(\widehat{x}_{i}, \widehat{x}_{-i}\right) \quad \text { for all } t_{i} \in S_{i} \text {. }
$$

This shows that $\widehat{x}=\left(\widehat{x}_{1}, \widehat{x}_{2}, \ldots, \widehat{x}_{n}\right)$ is a generalized Nash equilibrium of this game. Similarly to the proof of Theorem 3.4, we can show that for any $\bar{z} \in[\widehat{x}], \bar{z}$ is also a generalized Nash equilibrium of this game. This completes the proof of this theorem.

Similar to Corollary 3.6, if we consider some special collections of strategies with lower bound, then we obtain the following consequence of Theorem 4.1, where condition (3.1)' can be removed.

Corollary 4.2 Let $\Gamma=(N, S, f, U)$ be an n-person nonmonetized noncooperative game. Suppose that for every player $i=1,2,3, \ldots, n, \wedge S_{i} \neq \varnothing$, and for any $x \in S, f_{i}$ satisfies assumptions g1 and g3. If $f_{i}$ satisfies one of assumptions $\mathrm{g} 2$ or $\mathrm{g} 2$ ' given in Theorem 4.1, then there is an $\succcurlyeq^{S}$-cluster in $S$, in which every element is a generalized Nash equilibrium of $\Gamma$. 
Competing interests

The authors declare that they have no competing interests.

\section{Authors' contributions}

Authors $L X$ and $J L$ initialed the basic ideas and definitions of this manuscript, and then all the three authors $L X, J L$ and $W Y$ worked together in proving all theorems.

\section{Author details}

${ }^{1}$ Department of Mathematics, Lishui University, Lishui, Zhejiang 323000, China. ${ }^{2}$ Department of Mathematics, Shawnee State University, Portsmouth, Ohio 45662, USA. ${ }^{3}$ Department of Mathematics, Zhejiang Normal University, Jinhua, Zhejiang 321000, China.

\section{Acknowledgements}

The authors are grateful to the anonymous reviewers for their valuable suggestions, which really improved the presentation of this paper. First author was partially supported by the National Natural Science Foundation of China (11171137). Third author was partially supported by the National Natural Science Foundation of China (Grant 11101379).

Received: 27 April 2013 Accepted: 8 July 2013 Published: 22 July 2013

\section{References}

1. Aubin, JP: Mathematical Methods of Games and Economic Theory. North-Holland, Amsterdam (1979)

2. Bohnenblust, HF, Karlin, S: Contributions to the Theory of Games. Princeton University Press, Princeton (1950)

3. Mas-Colell, A, Whinston, MD, Green, JR: Microeconomic Theory. Oxford University Press, Oxford (1995)

4. Samuelson, P. Foundations of Economic Analysis. Harvard University Press, Cambridge (1947)

5. Zhang, CJ: Set-Valued Analysis with Applications in Economics. Sciences Press, Beijing (2004) (Chinese)

6. Von Neumann, J, Morgenstern, O: The Theory of Games and Economic Behavior. Princeton University Press, Princeton (1944)

7. Carl, S, Heikkilä, S: Fixed Point Theory in Ordered Sets and Applications: From Differential and Integral Equations to Game Theory. Springer, New York (2010)

8. Li, JL: Extended and generalized Nash equilibria of nonmonetized noncooperative games on complete lattices (submitted)

9. Li, JL: Applications of fixed point theory to generalized Nash-equilibriums of nonmonetized noncooperative games on Banach lattices. Nonlinear Anal. Forum 18, 1-11 (2013)

10. Li, JL: Extended Nash equilibria of nonmonetized noncooperative games on preordered sets. Int. Game Theory Rev. (to appear)

11. Li, JL, Park, S: Generalized Nash-equilibriums of nonmonetized noncooperative games on lattices. Br. J. Econ. Manag. Trade (to appear)

12. Li, JL: Several extensions of the Abian-Brown fixed point theorem and their applications to extended and generalized Nash equilibria on chain-complete posets. J. Math. Anal. Appl. (to appear)

13. Xie, LS, Li, JL, Wen, CF: Applications of fixed point theory to extended Nash-equilibriums of nonmonetized noncooperative games on posets (to appear)

14. Ok, EA: Order theory (forthcoming)

15. Fujimoto, T: An extension of Tarski's fixed point theorem and its applications to isotone complementarity problems. Math. Program. 28, 116-118 (1984)

16. Aliprantis, CD, Burkinshaw, O: Positive Operators. Springer, Berlin (2006)

17. Dunford, N, Schwartz, JT: Linear Operators. Part I. Wiley, New York (1988)

doi:10.1186/1687-1812-2013-192

Cite this article as: Xie et al.: Order-clustered fixed point theorems on chain-complete preordered sets and their applications to extended and generalized Nash equilibria. Fixed Point Theory and Applications 2013 2013:192.

\section{Submit your manuscript to a SpringerOpen ${ }^{\circ}$ journal and benefit from:}

- Convenient online submission

Rigorous peer review

- Immediate publication on acceptance

- Open access: articles freely available online

- High visibility within the field

- Retaining the copyright to your article 